\title{
A Population-Based Threshold of Protection for COVID-19 Vaccines
}

\section{David Goldblatt ( $\nabla$ d.goldblatt@ucl.ac.uk)}

Great Ormond Street Institute of Child Health Biomedical Research Centre University College London https://orcid.org/0000-0002-0769-5242

\section{Andrew Fioré-Gartland}

Fred Hutchinson Cancer Research Center https://orcid.org/0000-0001-7627-2166

\section{Marina Johnson}

University College London

\section{Adam Hunt}

University College London

\section{Christopher Bengt}

University College London

\section{Dace Zavadska}

4) Children's Clinical University Hospital

\section{JEREMY BROWN}

UNIVERSITY COLLEGE LONDON https://orcid.org/0000-0002-5650-5361

\section{Lesley Workman}

University of Cape Town

\section{Heather Zar}

University of Cape Town

\section{David Montefiori}

Duke University

\section{Xiaoying Shen Shen}

Duke University Medical Center

\section{Peter Dull}

Bill and Melinda Gates Foundation

\section{Stanley Plotkin}

University of Pennsylvania

\section{George Siber}

Independent Advisor

\section{Donna Ambrosino}

Independent Advisor 
Article

Keywords: COVID-19, vaccines, mRNA, vaccine efficacy

Posted Date: August 31st, 2021

DOl: https://doi.org/10.21203/rs.3.rs-832531/v1

License: (c) (i) This work is licensed under a Creative Commons Attribution 4.0 International License. Read Full License 


\section{Abstract}

Correlates of protection for COVID-19 vaccines are urgently needed to license and deploy additional vaccines. We measured immune responses to four COVID-19 vaccines of proven efficacy using a single serological platform calibrated to the international standard. IgG anti-Spike antibodies correlated significantly with efficacies for original virus and alpha variant and were highly correlated with ID50 neutralization in a validated pseudoviral assay. The protective threshold for each vaccine was calculated for IgG anti-Spike antibody. The mean protective threshold for all vaccine studies was $154 \mathrm{BAU} / \mathrm{ml}$ (95\% Cl 42-559), and for the vaccine studies with antibody distributions that enabled precise estimation of thresholds (i.e. leaving out 2-dose mRNA studies) was $60 \mathrm{BAU} / \mathrm{ml}(95 \% \mathrm{Cl} 35-102)$. We propose that the proportion of individuals with responses above the appropriate protective threshold together with the geometric mean concentration can be used in comparative non-inferiority studies with licensed vaccines to ensure that new vaccines will be efficacious.

\section{Introduction}

Severe acute respiratory syndrome-related coronavirus-2 (SARS-CoV-2) was first recognised in December 2019 and rapidly spread world-wide resulting in WHO declaring a COVID-19 pandemic on March 11th, 2020. Soon after the identification and genetic sequencing of the virus, numerous groups began developing vaccines with unprecedented speed and using a variety of approaches. Randomized controlled efficacy trials have shown a range of efficacies and have supported emergency use authorizations of more than eleven vaccines. In high- income countries the majority of vaccines distributed are those from Pfizer/BioNTech, Moderna, AstraZeneca and Johnson and Johnson. Numerous additional vaccines are in development but confirming their efficacy in randomized placebo-controlled trials is becoming increasingly difficult. Given the urgent need for additional vaccines to meet the global demand, licensing new vaccines based on serologic correlates of protection is of critical importance.

Two recent studies have shown strong correlations between both neutralizing antibodies and IgG binding antibodies with protective efficacy in clinical efficacy trials. Indeed, up to $90 \%$ of the variability in efficacy observed among different vaccines which used different technology platforms could be explained by their antibody levels, suggesting that post-immunization antibody levels can serve as a valid measure of short-term protection ${ }^{1,2}$. Of note, in the absence of standardized antibody assays needed to support comparisons between trials, both studies had to rely on normalizing the published antibody levels to a "best available" standard, the antibody levels in human convalescent serum measured in the corresponding assays.

An immunological correlate of protection (COP) is an invaluable tool for developing and deploying new vaccines, as it enables evaluation of vaccine candidates based on immunological endpoints. In practice, a COP has been established for many licensed vaccines based on a protective threshold or minimum protective level $^{3}$. Two main methods have been used: individual-based correlates and population-based correlates (WHO/IVB/13/01). The individual-based correlate measures biomarkers prior to exposure in all 
vaccinated subjects and evaluates the relationship between these and the development of disease. The expectation is that a concentration of the relevant biomarker (most commonly a level of antibody) can be found above which individuals are reasonably likely to be protected. This method has been applied to a number of diseases such as measles ${ }^{4,5}$ and meningococcus ${ }^{6}$, typically by evaluating outbreaks of disease in which, fortuitously, pre-outbreak sera were available. The method has rarely been used in largescale vaccine trials because of the inconvenience and expense of collecting sera on all participants, but some COVID-19 vaccine trials are an exception. Indeed, the individual-based method has very recently been applied to the AstraZeneca and Moderna COVID-19 vaccine trials, both of which showed that spikespecific antibody binding is associated with lower risk of symptomatic disease, but a threshold above which subjects were reliably protected could not be identified 7,8 .

The population-based approach conceived by Chang and Kohberger requires the measurement of antibody in a representative sample of subjects after vaccination and calculates the protective threshold based on the observed efficacy by using the simplifying assumption that all subjects with antibody above the threshold are fully protected and all subjects below the threshold are fully at risk of disease ${ }^{9}$. This method has been applied to meningococcal $\mathrm{C}$ vaccine using post-licensure efficacy data in England ${ }^{10}$ and pneumococcal vaccines based on multiple efficacy trials ${ }^{11}$. Protective thresholds identified by this method have been widely accepted by regulatory authorities and have proved useful for licensing multiple follow-on vaccines. This method does not rely on measurement of antibodies in individuals who have breakthrough infections but rather on defining the distribution of antibodies in a representative subset of the immunized population and hence is referred to as a population-based analysis.

A prerequisite for estimating a broadly applicable COP is an antibody assay, using similar or identical protocols, which has been shown to give equivalent results in different laboratories as urged by many including the CDC COVID-19 Response Team ${ }^{12}$. A WHO standard polyclonal antibody is now available, which was used here to normalize antibody levels in Binding Antibody Units (BAU) ${ }^{13}$. To date, very few studies comparing immunogenicity between vaccines have been undertaken although regulators are now beginning to request that direct comparisons be made between new vaccines in development and vaccines with demonstrated efficacy (https://valneva.com/press-release/valneva-initiates-phase-3clinical-trial-for-its-inactivated-adjuvanted-covid-19-vaccine-candidate-vla2001). In such studies it will be important to compare immune responses based on the proportion of participants with antibody responses above the protective threshold and the geometric means, both of which have been used previously to compare vaccine immunogenicity in pivotal licensure studies.

In this study, we collected samples of serum obtained from vaccinees who received 1 or 2 doses of 4 vaccines: BNT162b (Pfizer), mRNA1273 (Moderna), ChadOx1/AZ 1222 (AstraZeneca), or Ad.26COV2.S $(\mathrm{J} \& \mathrm{~J})$, and compared the IgG responses to the spike antigen, RBD antigen, and ACE2 Receptor blocking activity to the original strain and the alpha variant (B.1.117). We then applied population-based methods to assess the correlation with vaccine efficacy or effectiveness and to estimate a protective threshold. Finally, we suggest a path toward formally establishing a correlate of protection for COVID-19 vaccines. 


\section{Results}

\section{Demographic Characteristics of the Four Vaccine Cohorts}

Serum samples from 122 adults following a complete immunization schedule of the 4 vaccines were available for analysis. In addition, serum samples from 83 of these individuals were available following a first dose of BNT162b (Pfizer), mRNA1273 (Moderna), and ChadOx1/AZ 1222 (AstraZeneca). Individuals with positive anti-Nucleocapsid antibody at the time of evaluation or history/evidence of prior COVID 19 infection were excluded from the analysis. The demographic characteristic of the four cohorts of naïve subjects are provided in Supplemental Table S1. The median age of the different cohorts was between 35-60 years with ages ranging from 21 to 77 years. For each individual cohort neither age nor gender were significantly associated with vaccine responses, so antibody responses of cohorts were compared without adjustments. For the vaccines authorised for two dose use, the median time between doses was 3 or 4 weeks for Pfizer, Moderna, and the interval for the AstraZeneca vaccine was 66 days reflecting the manner of its deployment in the United Kingdom where the vaccinees resided.

\section{Comparative IgG Spike and RBD Antibody to Original and Alpha Variant for Four Vaccine Cohorts}

IgG binding antibody concentrations to the original virus (with D614G) and B.1.1. 7 (alpha variant) Spike and RBD antigens were assessed by ELISA and expressed as Binding Antibody Units/ml (BAU/ml) for each cohort utilizing the WHO standard as detailed in Methods. The alpha variant was the first SARSCoV-2 variant designated by WHO as a Variant of Concern (VOC) with significantly increased transmissibility and possibly increased severity of illness-

The comparative responses to both original virus and alpha variant Spike and RBD are shown in Figure 1. The geometric mean concentrations (GMC) to S-original antigen following Moderna and Pfizer vaccines were $4552 \mathrm{BAU} / \mathrm{ml}$ and $2468 \mathrm{BAU} / \mathrm{ml}$ respectively compared to $196 \mathrm{BAU} / \mathrm{ml}$ and $61 \mathrm{BAU} / \mathrm{ml}$ following AstraZeneca and J\&J vaccines respectively. A Kruskal Wallis test of GMC demonstrated significant differences for all groups. Each cohort had a reduced GMC for S-alpha compared to S-original ranging from 1.17-1.78-fold reduction. Similar differences of GMC between cohorts were noted for antibody to the S-alpha as for S-original. The results for ELISA antibody directed to RBD were similar to ELISA antibody to Spike with Pfizer, Moderna vaccine cohorts demonstrating between 5-105 higher GMC compared to AstraZeneca and J\&J cohorts for both the original virus and alpha variant. Of note, the assignment of units for the WHO standard for Spike and RBD ELISA were the same at $1000 \mathrm{units} / \mathrm{ml}$ and thus values do not represent absolute amount of antibody directed to-Spike compared to RBD.

\section{Comparative ACE-2 Inhibition Assay Results for Original and Alpha Variant for Four Vaccine Cohorts}

ACE-2 is the protein expressed on human cells that is the receptor for RBD of CoVID-19 virus with attachment required for entry into cells. Antibody directed to the virus that interferes with the viral receptor interaction results in neutralization and is proposed as a primary mechanism of protection from infection. 
Assays to assess neutralization are difficult to standardize and are slow through-put and thus measurement of ACE-2 inhibition by an ELISA format has been developed. Results using this assay have

also been demonstrated to correlate with neutralization assay readouts in several studies ${ }^{14,15}$. We therefore examined the ACE-2 Inhibition of antibody directed to both Spike and RBD antigens of original and alpha variant for each of the cohorts. Data are shown for original and alpha variant in Supplementary Figure S1 for Spike and RBD. The relative amounts of inhibition for each cohort to Spike and RBD demonstrate the same hierarchy of responses as were demonstrated for direct binding IgG Spike and RBD antibody assays.

\section{Correlation of ELISA IgG Spike, RBD and ACE-2 Inhibition to Neutralization Assays}

To further define the relationship of ELISA binding antibody and inhibition to neutralization, we assayed a subset of 75 sera in a formally validated neutralization assay with lenti-pseudovirus in the laboratory of David Montefiori. We examined the correlation to ID 50 neutralization with D614G pseudovirus for IgG binding antibody to Spike and RBD and inhibition of ACE-2 to Spike, and inhibition of ACE-2 to RBD of original virus. As displayed in Figure 2, correlations were observed for all the comparisons with significant correlation coefficients ranging from 0.73 to 0.90 .

\section{Correlation of GMC IgG Spike Antibody with Vaccine Efficacy Across Four Vaccines}

We and others found a remarkably high correlation between ELISA binding antibody or neutralizing antibody and efficacy across the different vaccines ${ }^{1,2}$. To be able to compare GM antibody levels measured by multiple assays and laboratories across the different trials both groups calibrated antibody levels relative to human convalescent antibodies in the same assay. In contrast, in this study we utilized a standardized, commercially available ELISA binding assay performed in a single laboratory to measure binding antibody elicited by different vaccines thereby circumventing the need for inter-laboratory assay standardization. We evaluated the correlation between GMC binding antibodies to Spike and RBD with the efficacy determined in Phase 3 studies or large recent effectiveness studies (Supplementary Table S3). Most efficacy and effectiveness trials were conducted when the original strain was predominant. A strong correlation was observed between antibody to original Spike antigen and efficacy with a rank correlation coefficient of 0.94 and with $97.4 \%$ of the variance explained by the antibody in a linear model (Figure 3A). Similar significant correlations were observed for GMC IgG antibody to the original RBD and efficacy (data not shown). Four of the vaccines in this study have reported clinical efficacy data for the alpha variant. The GMC IgG antibody to alpha variant Spike and vaccine efficacy against alpha variants showed a rank correlation coefficient of 0.83 with $68.6 \%$ of the variance explained by the antibody in a linear model (Figure 3B). 


\section{Estimation of a Protective Threshold for Each Vaccine Regimen Using Reverse Cumulative Distribution (RCD) Curves}

The high correlation between vaccine efficacy and the binding antibody to Spike and RBD together with the high correlation of these binding antibodies with a functional pseudovirus neutralization assay provide a strong rationale to evaluate binding assays to estimate the protective threshold for COVID-19 vaccines. The purpose of the protective threshold, sometimes called the minimum protective level of antibody, is to define the cut-off between antibody levels deemed sufficient to provide protection in a population and those which are not deemed protective. Unlike the GMC of antibody, which has been shown to vary with vaccine efficacy, we expect the protective threshold to be similar for vaccines demonstrating different levels of efficacy, provided that factors affecting the amount of antibody needed for protection of the populations as a whole are similar among studies, including quality of antibody and other immune mechanisms, clinical endpoint, intensity of exposure and vaccine strain. The population of subjects with antibody levels above the threshold are expected to be protected and those with antibody levels below the threshold are expected to be at risk, regardless of which vaccine was given.

We estimated protective thresholds for each vaccine using the population-based model developed by Chang and Kohberger for pneumococcal vaccine ${ }^{9}$. The model makes one critical simplifying assumption which is that there is a sharp cut-off between protective and non-protective antibody levels: i.e. that all individuals, whether immunized or controls, with antibody above the threshold are protected and those with antibody below it are at risk. In the studies evaluated here control subjects had no pre-existing antibody so the threshold can simply be determined from the post-immunization RCD curves, such that the percent of subjects above the threshold is equal to the percent efficacy observed.

The RCD curves were estimated from immune responses to vaccine regimens for which efficacy estimates were available, including one or two doses of Pfizer, Moderna, and AstraZeneca vaccines, and one dose of J\&J. (Figure 4). When all vaccines and dose regimens are included, the overall protective threshold was estimated to be $154 \mathrm{BAU} / \mathrm{ml}(95 \% \mathrm{Cl} 42-559)$ by taking the geometric mean of all thresholds across all six regimens using a random effects meta-analysis approach. A similar overall protective threshold was estimated for the alpha variant at $171 \mathrm{BAU} / \mathrm{ml}(95 \% \mathrm{Cl} ; 57-519)$ (Figure 5).

The thresholds calculated from the 2-dose mRNA vaccine regimens were outliers among the vaccine regimens evidenced by the lack of overlap between $95 \%$ confidence intervals of the Moderna threshold and the average threshold (Figure 5). It is also evident in Figure 1 that neither 2-dose mRNA regimen induces antibody levels below the mean threshold of $154 \mathrm{BAU} / \mathrm{ml}$ and thus do not contribute information to estimating a mean threshold that would be useful in comparing efficacious vaccines with lower immunogenicity. Therefore, another analysis was performed on less immunogenic but clearly efficacious vaccines by omitting the 2-dose mRNA vaccine data (but retaining the 1-dose mRNA vaccine data). The 
mean threshold excluding the two-dose mRNA vaccine data was $60 \mathrm{BAU} / \mathrm{ml}$ (95\% $\mathrm{Cl} 35-102)$. This lower value may be better suited for comparing vaccines which have lower but useful efficacy.

Finally, to verify that the threshold indeed discriminates between protected and non-protected populations in each study, we estimated the proportion of participants receiving each vaccine regimen that responded with antibody greater than the higher threshold of $154 \mathrm{BAU} / \mathrm{mL}$ (Figure S2A) or the lower threshold of 60 $\mathrm{BAU} / \mathrm{ml}$ (Figure S2B). In the figures, the line of identity indicates that under a model of an absolute protective threshold, efficacy would be expected to be equal to the proportion of participants above the threshold; the mean squared error from the line of equivalence is a useful metric for evaluating this relationship. The data support that there is an association between vaccine efficacy and the proportion of individuals above either of the thresholds. In practice a threshold could be used to directly compare two vaccines using a non-inferiority margin. It is noteworthy that for OX/AZ1 and J\&J1 vaccines the proportion of subjects above the lower threshold of $60 \mathrm{BAU} / \mathrm{ml}$ is quite similar to their efficacy, whereas that is not the case with the higher threshold of $154 \mathrm{BAU} / \mathrm{ml}$.

\section{Discussion}

Immunologic correlates of protection have played a critically important role in vaccine development by providing a framework for comparing newly developed vaccines to those which have already been approved based on demonstration of clinical efficacy. Such correlates are also needed for confirming consistent immunogenicity in initial lot-to-lot consistency trials, after major manufacturing changes of approved vaccines or in evaluating reduced doses of vaccines ${ }^{16}$. Multiple lines of evidence including preclinical studies and strong correlations between antibody levels and clinical efficacy after multiple vaccines show that antibodies are a major protective mechanism against primary infection with COVID$19^{1,2}$. Nevertheless, as of this writing, an antibody level associated with protection has not been defined for COVID-19 vaccines even though more than 10 large scale efficacy trials demonstrating a range of efficacies have been completed. Key reasons for this include the lack of well standardized antibody assays that facilitate direct comparisons between vaccines and lack of agreement on the methodology to be used to establish a protective correlate. Here we propose to overcome these obstacles by directly comparing immune responses of four vaccines in the same antibody assays in a single laboratory and by using an accepted population-based approach to estimate a threshold of protection based on observed vaccine efficacies in clinical trials.

The underlying assumptions of the population-based approach is that vaccines that induce antibodies above a protective threshold in a similar proportion of subjects can be expected to have similar clinical efficacy. A second assumption is that the protective threshold in a population can be modelled as a single cut-off concentration above which everyone is protected and below which everyone is at risk. Like all models, this is a gross simplification of the reality that individuals may require differing levels of antibody for protection for many reasons including host factors, pathogen virulence and intensity of exposure. The population-based protective threshold can be estimated in each trial from the observed efficacy and the antibody distribution several weeks after immunization, without further assumptions. With other 
vaccines such as pneumococcal and meningococcal vaccines, the threshold of protection was estimated by this method and was subsequently used as the primary outcome in numerous vaccine non-inferiority comparisons. The protective threshold provides the most sensitive ability to discriminate differences in immunogenicity ${ }^{10,11}$ and supports the use of a narrow non-inferiority margin for vaccines with very high efficacy e.g. $10 \%$ was recommended by WHO for pneumococcal conjugate vaccines ${ }^{17}$. Geometric mean titer or concentration can also serve as important outcomes but typically require comparative ratios of 1.5 to 2 -fold to exclude meaningful differences.

Estimates of the population-based threshold of protection are expected to be similar in each efficacy study, regardless of the efficacy that was observed, with the caveat that certain systematic differences among studies such as the quality of antibody induced, outcome definition, incidence of disease, strain causing disease or contribution of other immunological responses such as $T$ cell responses may affect the amount of antibody needed for protection at a population level. For example, a range of estimates for thresholds for anti-polysaccharide antibodies were determined for the pneumococcal vaccine but a single consensus estimate of $0.35 \mathrm{ug} / \mathrm{ml}$ was agreed upon as a protective threshold at a WHO Workshop attended by key stakeholders which has proven very useful in facilitating the approval of five additional vaccines around the world. Post-introduction effectiveness studies of these vaccines have confirmed their efficacy.

In this study the mean protective threshold for the original strain, based on data from all six vaccine regimens was $154 \mathrm{BAU} / \mathrm{ml}$ (95\% $\mathrm{Cl} 42-559)$. Because two doses of mRNA vaccines induced extremely high antibody levels, estimated protective thresholds with these two regimens had little or no overlap with those in the remaining five vaccine trials including one-dose mRNA regimens (Figure 5). We therefore calculated a mean threshold omitting the 2-dose mRNA data which was $60 \mathrm{BAU} / \mathrm{ml}$. with a narrower $95 \%$ $\mathrm{Cl}$ of 35-102 BAU/ml. The use of this lower threshold may be more appropriate for comparing new COVID-19 vaccines in non-inferiority trials against approved vaccines other than Pfizer and Moderna mRNA vaccines.

Recently, concentrations of neutralizing antibody associated with protection were estimated after the AstraZeneca and Moderna vaccines using a different approach based on how well post-immunization antibody levels predicted risk of acquiring COVID-19 for the individual subjects in their efficacy studies ${ }^{7,8}$. Both studies found broad overlap in antibody concentrations between individuals who developed COVID and those who did not. Consequently, it was not possible to identify a threshold antibody level above which subjects were reliably protected using analysis of breakthrough cases. Interestingly, however, Feng et al estimated a level of spike binding $\mathrm{IgG}$ antibody of $264 \mathrm{BAU} / \mathrm{ml}$ associated with $80 \%$ protection ${ }^{7}$ by the AstraZeneca vaccine and Gilbert et al, estimated a level of $298 \mathrm{BAU} / \mathrm{ml}$ associated with $90 \%$ protection by the Moderna vaccine ${ }^{8}$. Although these estimates were not threshold values, they are in a similar range to our population-based method for determining a threshold value.

The goal of developing a threshold of protection is to serve as the primary outcome for non-inferiority studies comparing new COVID-19 vaccines to vaccines already approved or authorized based on clinical 
efficacy. To accomplish this goal, the following key issues and questions should be resolved: First, which assays can be used as the primary basis for comparison? Binding assays have significant advantages in reproducibility, lower variability, cost and convenience and have been much easier to standardize among laboratories. Given that for COVID-19 various binding and neutralization assays show a high degree of correlation with each other and both are highly correlated with clinical protection, it is reasonable to propose that binding assays can serve as the primary serologic outcome in vaccine comparisons. Interestingly though, virus neutralization is difficult to demonstrate after one dose of mRNA vaccines despite efficacy up to $81 \%$ measured from $14 \mathrm{~d}$ after the first dose (https://www.pitchstudy.org/PITCH_Dosing_Interval_23072021.pdf). In contrast, binding antibody responses assessed following one dose of mRNA vaccines are easily measured and even superior to some less immunogenic vaccines after two doses. A plausible explanation for this discordance between binding and neutralizing antibodies is that the first dose of mRNA vaccine activates a non-neutralizing recall response predominantly targeting epitopes in the $\mathrm{S} 2$ subunit which is highly conserved across human pathogenic coronaviruses $^{18}$. Second, a consensus should be obtained on the threshold level to be used for noninferiority studies and the level of non-inferiority that must be demonstrated. Based on our analyses in this study, a protective threshold of $60 \mathrm{BAU} / \mathrm{ml}$ would provide an appropriate basis for non-inferiority comparisons of vaccines when compared against existing approved vaccines. A level of $154 \mathrm{BAU} / \mathrm{ml}$ may be a conservative estimate of the protective threshold and would provide a substantial "cushion" to guarantee efficacy but may prove difficult to use successfully for non-inferiority comparisons against approved vaccines with lower immunogenicity than the current mRNA vaccines. Third, the ongoing evolution of variants of concern (VOC) poses a critical question how protective thresholds can be estimated for VOCs. In this report, we used a population-based model to calculate threshold values for the alpha variant based on IgG antibody responses to alpha spike after the original strain vaccines and three efficacy estimates. The mean protective threshold to the alpha VOC was $171 \mathrm{BAU} / \mathrm{ml}$ which is in a similar range as the original strain. It is not yet known whether other VOCs which are more highly transmissible such as delta ${ }^{19}$ will also have similar protective levels or require higher levels of antibody for protection. The population-based method could readily be applied to other outcomes such as severe disease or asymptomatic infection but we could not find sufficient data to do these analyses. It would be extremely desirable to soon reach a consensus among stake holders on the appropriate protective threshold and non-inferiority requirements to facilitate approval of the numerous COVID-19 vaccines in the pipeline.

Limitations of our study include that a limited number of sera were assayed to construct the RCD curves, that our subjects may not be representative of those in the phase 3 efficacy studies and that only short term efficacy data are available. It would therefore be important to confirm the threshold estimates using larger numbers of sera from subjects enrolled in efficacy trials. It is noteworthy that the recent report by Gilbert et al estimated a Spike IgG threshold of $77 \mathrm{BAU} / \mathrm{ml}(95 \% \mathrm{Cl} 60-94)$ using the population-based method on a large serum set drawn on day 29 after the $1^{\text {st }}$ dose of the Moderna vaccine Phase 3 study ${ }^{8}$. When applied to sera after the $2^{\text {nd }}$ dose, the threshold estimate was $1000 \mathrm{BAU} / \mathrm{ml}(95 \% \mathrm{Cl} 860-1200)$ clearly demonstrating that the population-based method is not applicable with vaccine regimens inducing antibodies levels far above the threshold. 
The population-based protective threshold for binding antibody proposed in this report could be useful not only to license new vaccines based on comparable immunogenicity but also to predict the need and timing for booster immunizations as concentrations of antibody wane. For example, if the Pfizer vaccine is confirmed to have reduced efficacy due to both waning antibody and predominance of delta variant in Israel, one could determine the threshold antibody concentrations associated with the reduced efficacy for the population at that time. Such a threshold could be applied to other populations to predict the proportion of the population at risk for that variant based on the percent of individuals waning below the determined threshold. A recent study evaluating antibody concentrations associated with breakthrough cases in Israel, showed that both neutralizing and IgG anti-Spike concentrations were lower in those cases compared to controls although a threshold value was not determined ${ }^{20}$.

In conclusion, we propose a threshold of protection for IgG antibody to original spike protein that would serve as a basis for comparing new vaccines to existing vaccines in non-inferiority studies. This protective level should be calculated from larger data sets from efficacious vaccines than those available to us and a consensus would need to be achieved on defining a single protective threshold. Although other studies of COP have focused on neutralization assays, we believe that the data within this report and other studies support the use of binding antibody which has many practical advantages ${ }^{21}$. Finally, the COP could be useful for supporting boosting recommendations as serum antibody concentrations wane and new virus variants cause rapid increases in the number of CoVID-19 cases.

\section{Methods}

This study was undertaken using a convenience sample obtained post vaccination from groups of individuals receiving vaccine as part of their government's national rollout campaigns. Samples from vaccinees in Latvia and South Africa were obtained as part of a previous study of HCWs in paediatric facilities $^{22}$ originally initiated at Great Ormond Street Hospital (COSTARS, IRAS 282713, ClinicalTrials.gov Identifier: NCT04380896). Ethics approval was obtained locally by the lead investigators of each site. In the UK, volunteers who were part of the COSTARS Study as well as others who had received vaccines as part of the government rollout altruistically agreed to donate serum to help evaluate an assay for measuring post vaccine immunity being run the UCL laboratory. Vaccinees received one of four vaccines depending on local use.

In Latvia and South Africa, serum was prepared and aliquoted, given a unique identifier locally and stored frozen until batch shipping to the WHO International Reference laboratory for Pneumococcal Serology at University College London, London, UK. Local UK samples had serum extracted and were stored frozen until batch tested. Samples were analyzed for the presence IgG to SARS-CoV-2 nucleocapsid protein as well as receptor binding domain of $\mathrm{S} 1$ and trimeric spike antigen derived from the original Wuhan strain as previously described ${ }^{23}$ as well as spike and RBD responses to VOCs B.1.1.7 (MSD ${ }^{\circledR}$ SARS-Coronavirus Plate 7, Rockville, MD). Alpha variant has the following mutations within the Spike protein which were all represented in the ELISA target Spike antigen (Spike Protein Substitutions: 69deletion, 70deletion, 144 deletion, E484K, S494P, N501Y, A570D, D614G, P681H, T716I, S982A, D1118, K1191N with not all alpha 
variants having the substitutions E484K, S494P, or K1191N). Responses to nucleocapsid protein as well as information about previous clinical symptoms compatible with COVID with or without tests confirming the diagnosis were used to stratify vaccinees into naïve or primed as this can influence antibody responses to the vaccine ${ }^{24}$. The MSD IgG assay was calibrated against the WHO international anti-SARSCov-2 antibody standard which assigns a value of 1000 Binding Antibody Units for the Spike, RBD and Nucleocapsid antigens. As no standards exist for the variants, the internal standard used was evaluated and adjusted for the variant antigens based on the binding signal obtained. For RBD, B1.1.7 binding was similar to original thus no adjustment was necessary.

The MSD assay was also used to evaluate ACE2 Receptor blockade against the relevant antigens highlighted above as previously described ${ }^{23}$.

Neutralization was measured in a formally validated assay that utilized lentiviral particles pseudotyped with SARS-CoV-2 spike and containing a firefly luciferase (Luc) reporter gene for quantitative measurements of infection by relative luminescence units (RLU). The assay was performed in 293T/ACE2.MF provided by Drs. Michael Farzan and Huihui Mu. Pseudoviruses were prepared in HEK293T/17 cells and titrated for infectivity in 293T/ACE2.MF cells as described ${ }^{25}$. For measurements of neutralization, a pre-titrated dose of pseudovirus (Wuhan-1 spike containing D614G) was incubated with 8 serial 5-fold dilutions of serum samples (1:20 starting dilution) in duplicate in a total volume of $150 \mu$ l for $1 \mathrm{hr}$ at 37oC in 96-well flat-bottom poly-L-lysine-coated culture plates. 293T/ACE2-MF cells were detached from T75 culture flasks using TrypLE Select Enzyme solution, suspended in growth medium $(100,000$ cells $/ \mathrm{ml})$ and immediately added to all wells $(10,000$ cells in $100 \mu \mathrm{L}$ of growth medium per well). One set of 8 wells received cells + virus (virus control) and another set of 8 wells received cells only (background control). After 66-72 hrs of incubation, medium was removed by gentle aspiration and $30 \mu \mathrm{l}$ of Promega $1 \mathrm{X}$ lysis buffer was added to all wells. After a 10-minute incubation at room temperature, 100 $\mu \mathrm{l}$ of Bright-Glo luciferase reagent was added to all wells. After 1-2 minutes, $110 \mu \mathrm{l}$ of the cell lysate was transferred to a black/white plate. Luminescence was measured using a GloMax Navigator luminometer (Promega). Neutralization titers are the inhibitory dilution (ID) of serum samples at which RLUs were reduced by either $50 \%$ (ID50) or $80 \%$ (ID80) compared to virus control wells after subtraction of background RLUs. Serum samples were heat-inactivated for 30 minutes at $56^{\circ} \mathrm{C}$ prior to assay.

\section{Statistical analysis}

Geometric mean titers were calculated for all groups of vaccinees and compared using a Kruskal Wallis test; groups were compared pairwise using non-parametric rank-sum tests. Empirical reverse cumulative distribution functions (RCDs) were estimated from the immune response datasets independently for each vaccine/regimen. The point-wise $95 \%$ confidence intervals (indicated by shaded regions in the figures) were constructed using the non-parametric "exact" method of Hutson ${ }^{26}$, based on fractional order statistics. To estimate a protective threshold for each regimen we imposed a model that assumes that all 
participants with an immune response above a specified level are protected ${ }^{11}$. Therefore, to estimate the protective threshold for a regimen we estimated the $V E^{\text {th }}$ quantile of the RCD, where $V E$ is either the associated vaccine efficacy or effectiveness. To capture the uncertainty in both the RCD and the estimate of VE we used a double bootstrap approach; In each $i^{\text {th }}$ iteration of the bootstrap we sampled $V E_{i}$ from a normal distribution with mean and variance that reflected the published data (on a log-VE scale). Then the $V E_{i}^{\text {th }}$ quantile was estimated from an RCD constructed from a resampling of the immune response data (fixed $n$, sampled with replacement). After 10000 iterations the $2.5^{\text {th }}$ and $97.5^{\text {th }}$ percentiles were estimated from the distribution of computed thresholds providing a $95 \%$ confidence interval (indicated by vertical dashed lines in the RCD figures). For the 1-dose mRNA dataset we combined data from the Pfizer and Moderna vaccines. A weighted RCD was created such that the distribution of immune responses reflected that of the cohort of individuals studied in Pilishvili et al. ${ }^{27}$ (23\% 1-dose of Moderna and 77\% 1dose of Pfizer), since that was the associated vaccine effectiveness used in downstream analysis. To accommodate the weights, the RCD 95\% Cl was computed using a bias-corrected and accelerated (BCA) bootstrap as opposed to the exact method that was employed for the other regimens. The protective threshold was estimated using the double bootstrap approach described above. To derive an overall protective threshold we used a random-effects meta-analysis of the estimated thresholds for each regimen. The model allows for each regimen to have its own true protective threshold, but provides an unbiased estimate of the geometric mean threshold given the uncertainty in estimating each individual threshold.

We conducted a population-level analysis to evaluate the association between vaccine efficacy/effectiveness (VE) and the distribution of immune responses. We call this a population-level analysis because we have estimates of VE and immune response and do not link immune responses to specific individuals in the VE studies, which is typical of individual-level analyses. The association of immune response with VE was assessed using a weighted least-squares linear regression. Both VE and immune responses were analyzed and fit after a log transformation. For the VE outcomes the weights were the inverse variances as published, which upweights studies with more precise estimates of VE. For immune response predictors we used a summary measure of the distribution of immune responses for each regimen; these summaries included estimates of: (i) geometric mean, or (ii) the proportion of participants above a fixed threshold (threshold estimated from the data using either all data or data excluding two-dose mRNA vaccines). Each of these was evaluated based on its ability to predict the observed VE, assessed using mean squared error (MSE). For the 1-dose mRNA responses each summary measure was computed by weighting the Pfizer and Moderna responses accordingly (see above). All analyses were conducted using Python3 with the numpy and statsmodels packages.

\section{Declarations}

Author Contributions 
All authors contributed to the design of the study, writing of the study and revision of the manuscript. Additionally, DG, JB, DZ, HZ, LW were responsible for sample collection, MJ, AH, CB, DM and XS were responsible for laboratory evaluation of samples and AFG for statistical analysis.

Competing Interests statement

The authors declare the following financial interests/personal relationships which may be considered as potential competing interests: Dr. Plotkin consults for Janssen and Moderna; Dr. Siber reports personal fees from Clover Biopharmaceuticals, personal fees from AdVaccine, other from Vaxxinity personal fees from CanSino, personal fees from CureVac, personal fees from Valneva, personal fees from Vaxart, personal fees and other from Affinivax, outside the submitted work; Dr. Ambrosino reports personal fees from Vaxxinity, personal fees from Clover Biopharmaceuticals, outside the submitted work.

\section{References}

1. Khoury, D.S., et al. Neutralizing antibody levels are highly predictive of immune protection from symptomatic SARS-CoV-2 infection. Nat Med 27, 1205-1211 (2021).

2. Earle, K.A., et al. Evidence for antibody as a protective correlate for COVID-19 vaccines. Vaccine 39, 4423-4428 (2021).

3. Plotkin, S.A. Correlates of protection induced by vaccination. Clin Vaccine Immunol 17, 1055-1065 (2010).

4. Chen, R.T., et al. Measles antibody: reevaluation of protective titers. J Infect Dis 162, 1036-1042 (1990).

5. Woudenberg, T., et al. Additional Evidence on Serological Correlates of Protection against Measles: An Observational Cohort Study among Once Vaccinated Children Exposed to Measles. Vaccines (Basel) 7(2019).

6. Goldschneider, I., Gotschlich, E.C. \& Artenstein, M.S. Human immunity to the meningococcus. I. The role of humoral antibodies. J Exp Med 129, 1307-1326 (1969).

7. Feng, S., et al. Correlates of protection against symptomatic and asymptomatic SARS-CoV-2 infection. medRxiv, 2021.2006.2021.21258528 (2021).

8. Gilbert, P.B., et al. Immune Correlates Analysis of the mRNA-1273 COVID-19 Vaccine Efficacy Trial. medRxiv, 2021.2008.2009.21261290 (2021).

9. Siber, G.R. Methods for estimating serological correlates of protection. Dev Bio/ Stand 89, 283-296 (1997). 
10. Andrews, N., Borrow, R. \& Miller, E. Validation of serological correlate of protection for meningococcal $\mathrm{C}$ conjugate vaccine by using efficacy estimates from postlicensure surveillance in England. Clin.Diagn.Lab Immunol. 10, 780-786 (2003).

11. Siber, G.R., et al. Estimating the protective concentration of anti-pneumococcal capsular polysaccharide antibodies. Vaccine $25,3816-3826$ (2007).

12. Gundlapalli, A.V., et al. SARS-CoV-2 Serologic Assay Needs for the Next Phase of the US COVID-19 Pandemic Response. Open Forum Infect Dis 8, ofaa555 (2021).

13. Kristiansen, P.A., et al. WHO International Standard for anti-SARS-CoV-2 immunoglobulin. Lancet 397, 1347-1348 (2021).

14. Case, J.B., et al. Neutralizing Antibody and Soluble ACE2 Inhibition of a Replication-Competent VSV-SARS-CoV-2 and a Clinical Isolate of SARS-CoV-2. Cell Host Microbe 28, 475-485 e475 (2020).

15. Tan, C.W., et al. A SARS-CoV-2 surrogate virus neutralization test based on antibody-mediated blockage of ACE2-spike protein-protein interaction. Nat Biotechno/ 38, 1073-1078 (2020).

16. Jurgens, G. Low Dose Regimens of BNT162b2 mRNA Vaccine Exceed SARS-Cov-2 Correlate of Protection Estimates for Symptomatic Infection, in those 19-55 Years of Age. medRxiv, 2021.2003.2006.21253058 (2021).

17. Jodar, L., et al. Serological criteria for evaluation and licensure of new pneumococcal conjugate vaccine formulations for use in infants. Vaccine 21, 3265-3272 (2003).

18. Brewer, R.C., Ramadoss, N.S., Lahey, L.J., Robinson, W.H. \& Lanz, T.V. BNT162b2 Vaccine Induces Divergent B cell responses to SARS-CoV-2 S1 and S2. medRxiv, 2021.2007.2020.21260822 (2021).

19. Li, B., et al. Viral infection and transmission in a large, well-traced outbreak caused by the SARSCoV-2 Delta variant. medRxiv, 2021.2007.2007.21260122 (2021).

20. Bergwerk, M., et al. Covid-19 Breakthrough Infections in Vaccinated Health Care Workers. N Eng/ J Med (2021).

21. Carreño, J.M., et al. Reduced neutralizing activity of post-SARS-CoV-2 vaccination serum against variants B.1.617.2, B.1.351, B.1.1.7+E484K and a sub-variant of C.37. medRxiv, 2021.2007.2021.21260961 (2021).

22. Goldblatt, D., et al. Cross-sectional prevalence of SARS-CoV-2 antibodies in healthcare workers in paediatric facilities in eight countries. J Hosp Infect 110, 60-66 (2021).

23. Johnson, M., et al. Evaluation of a novel multiplexed assay for determining IgG levels and functional activity to SARS-CoV-2. J Clin Viro/ 130, 104572 (2020). 
24. Krammer, F., et al. Antibody Responses in Seropositive Persons after a Single Dose of SARS-CoV-2 mRNA Vaccine. N Engl J Med 384, 1372-1374 (2021).

25. Shen, X., et al. SARS-CoV-2 variant B.1.1.7 is susceptible to neutralizing antibodies elicited by ancestral spike vaccines. Cell Host Microbe 29, 529-539 e523 (2021).

26. Hutson, A.D. Calculating nonparametric confidence intervals for quantiles using fractional order statistics. Journal of Applied Statistics 26, 343-353 (1999).

27. Pilishvili, T., et al. Interim Estimates of Vaccine Effectiveness of Pfizer-BioNTech and Moderna COVID-19 Vaccines Among Health Care Personnel - 33 U.S. Sites, January-March 2021. MMWR Morb Mortal Wkly Rep 70, 753-758 (2021).

\section{Table}

Table 1: Geometric Mean Concentrations (GMC) to Spike of original virus and alpha variant following complete course of immunization. IgG GMCs and $95 \% \mathrm{Cl}(\mathrm{BAU} / \mathrm{ml})$ to Spike derived from original (D614G) as well as the B.1.1.7 (Alpha Variant), following complete courses of four different SARS CoV 2 vaccines administered to naïve recipients. GMCs are expressed in standardised Binding Antibody Units (BAU) /ml calibrated against the WHO international standard. A Kruskal Wallis test demonstrated significant difference between groups and pair wise comparisons using a rank based test demonstrated $p<0.001$ for all groups except for Moderna compared to Pfizer at $p<.007$, and J\&J compared to AstraZeneca at $p<0.002$.

\begin{tabular}{|lllll|}
\hline Vaccine & Manufacturer & $\begin{array}{l}\text { GMC Original } \\
\text { Spike } \\
\text { BAU/ml (95\% } \\
\text { Cl) }\end{array}$ & $\begin{array}{l}\text { GMC Alpha Variant } \\
\text { Spike } \\
\text { BAU/ml (95\% Cl) }\end{array}$ & $\begin{array}{l}\text { Fold Reduction of } \\
\text { Original } \\
\text { Compared to } \\
\text { Alpha }\end{array}$ \\
\hline mRNA 1273 & Moderna & $\begin{array}{l}4552(3621- \\
5273)\end{array}$ & $3888(2835-5331)$ & 1.17 \\
\hline BNT162b & Pfizer & $\begin{array}{l}2468(1983- \\
3072)\end{array}$ & $1736(1363-2211)$ & 1.46 \\
\hline $\begin{array}{l}\text { ChadOx/AZD } \\
\text { 1222 }\end{array}$ & AstraZeneca & $196(140-273)$ & $108(76-154)$ & 1.78 \\
\hline Ad.26COV2.S & J\&J & $61(37-101)$ & $37(22-62)$ & 1.65 \\
\hline
\end{tabular}

\section{Figures}


a)

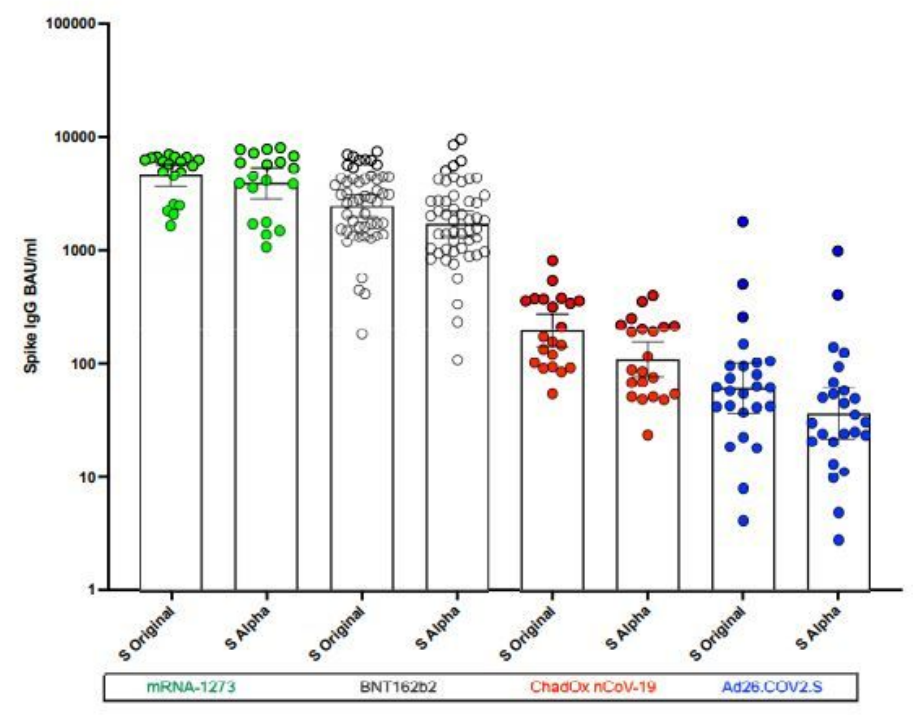

b)

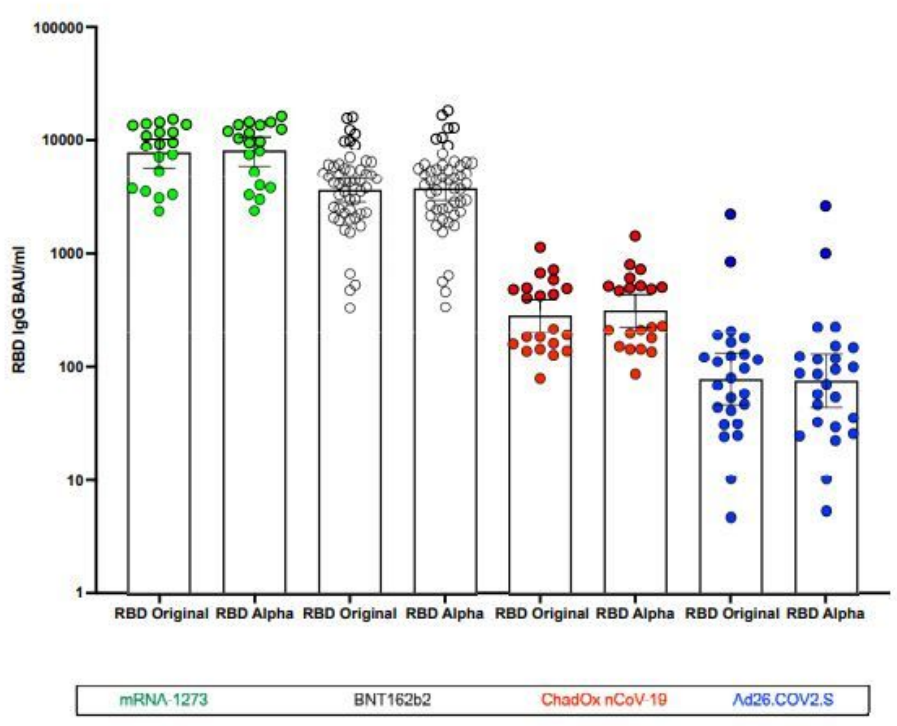

\section{Figure 1}

Spike and RBD IgG Antibody to original virus and alpha variant for fully immunized cohorts. a) to Spike b) to RBD. IgG Concentrations, GMCs and $95 \% \mathrm{Cl}(\mathrm{BAU} / \mathrm{ml})$ to Spike and RBD derived from Original Virus as well as the B.1.1.7 (Alpha Variant), following complete courses of four different SARS CoV 2 vaccines administered to naïve recipients. Concentrations are expressed in standardised Binding Antibody Units $(\mathrm{BAU}) / \mathrm{ml}$ calibrated against the WHO international standard, GMC are displayed by bar with $95 \% \mathrm{Cl}$ represented by whiskers. 
a
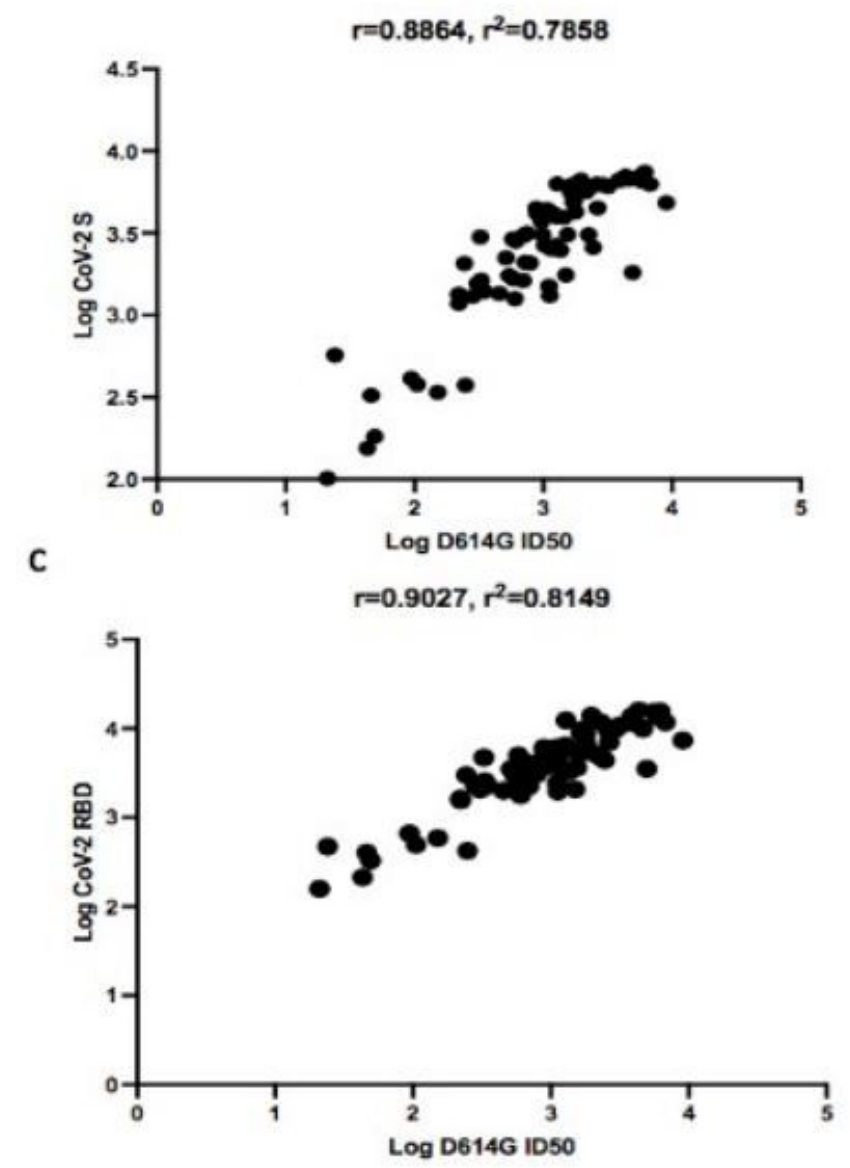

b
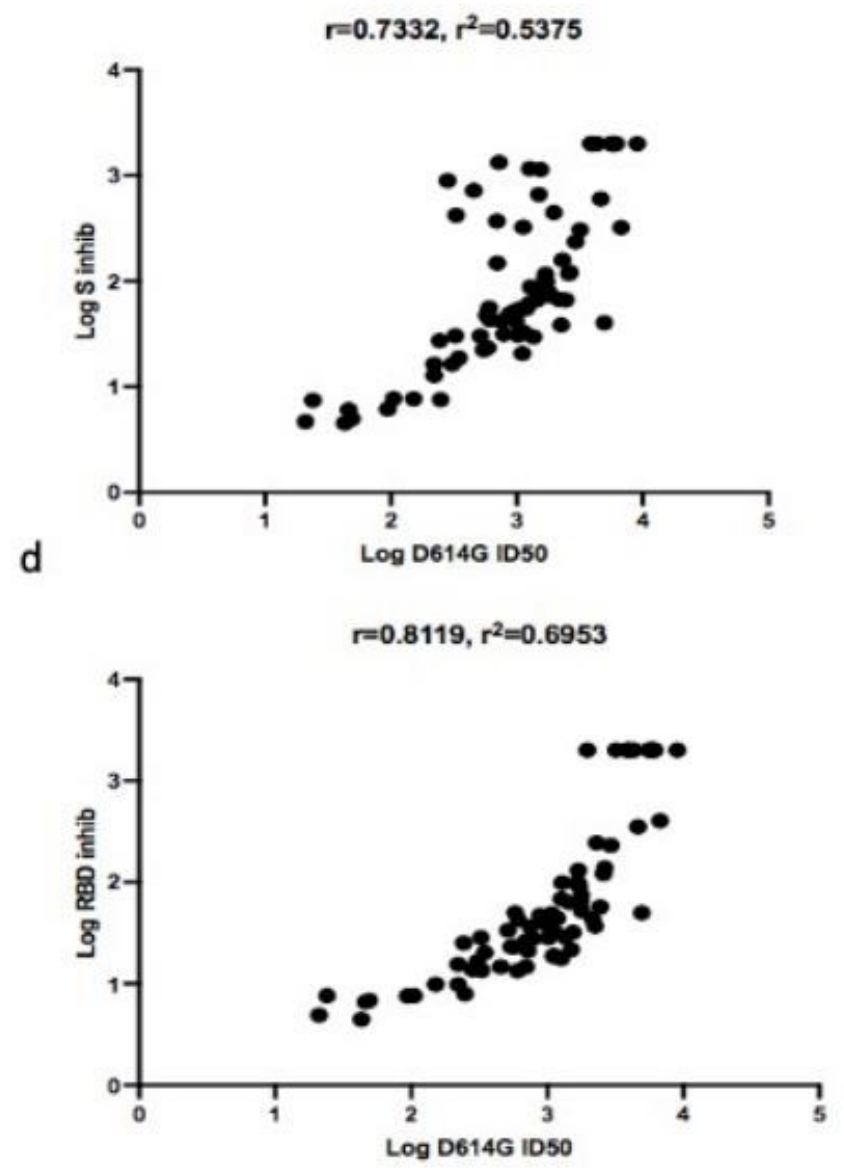

Figure 2

Correlations of ELISA binding antibody and ACE-2 inhibition to neutralization assay with D614G pseudovirus: For a subset of samples from multiple vaccine cohorts ID 50 neutralization was assayed with D614G pseudovirus and correlated to a) antibody to Spike b) antibody to RBD c) ACE-2 inhibition of Spike and d) ACE-2 inhibition of RBD. A rank correlation value was calculated at $r=0.89,0.90,0.73$, and 0.81 respectively and $r 2$ in a linear model was utilized for variance explanations. 
A

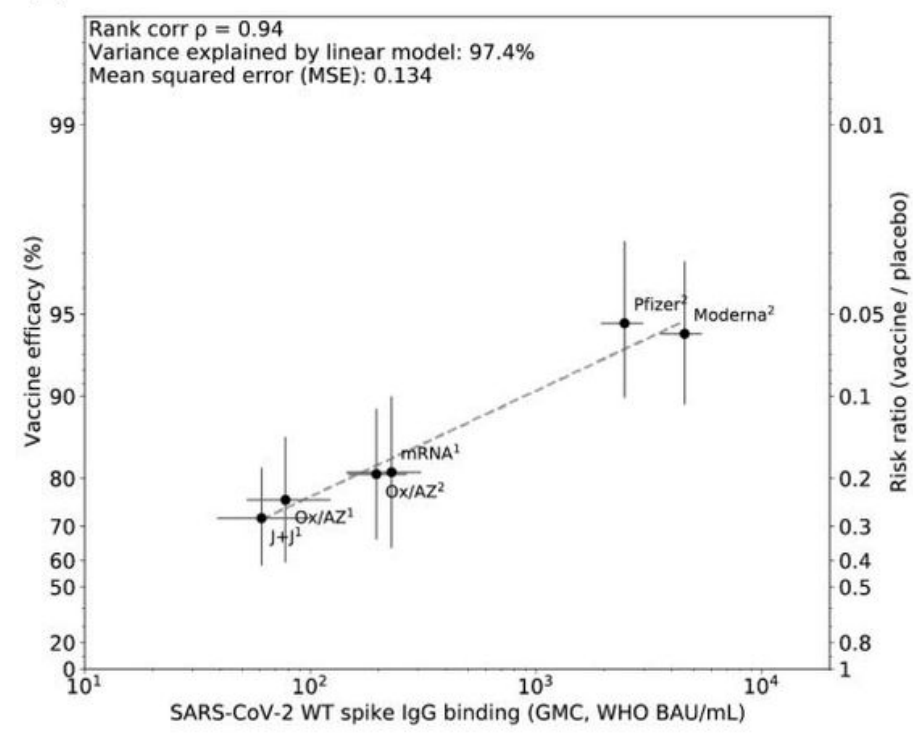

B

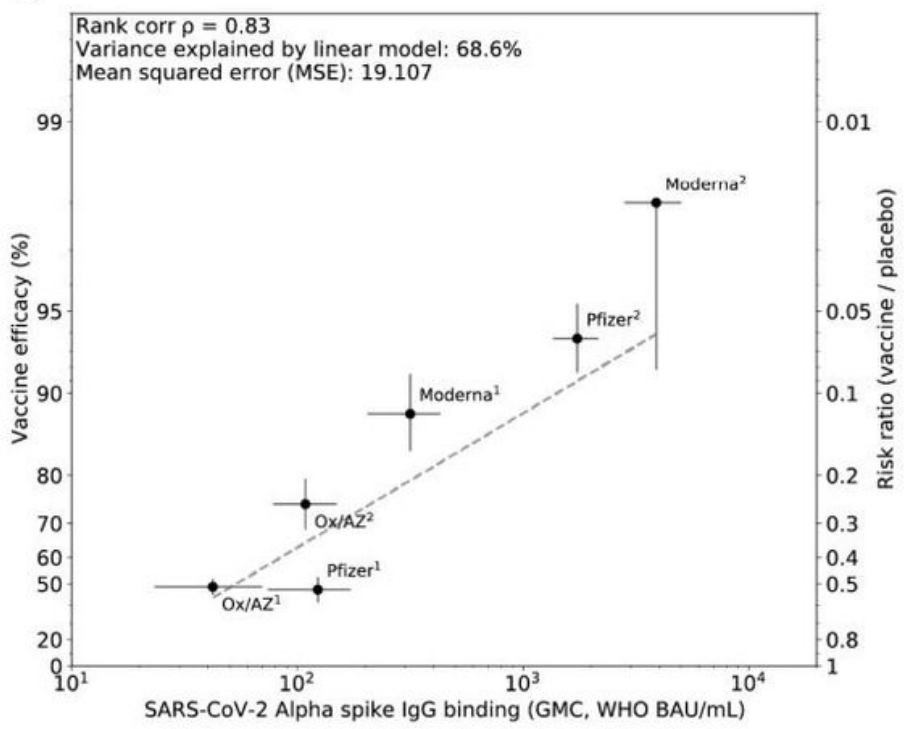

Figure 3

Correlation of Spike IgG antibody binding with vaccine efficacy. Vaccine efficacy/effectiveness (VE) and SARS-CoV-2 spike binding IgG, both against either an (A) Original (WT) strain virus (for VE) and spike antigen (for $\lg G$ ) or (B) an Alpha strain virus and spike antigen. Data included in correlation analyses are described in Table 1 and Table S2. Superscript 1 or 2 indicates the number of doses for the vaccine regimen. The $y$-axis is estimated log risk-ratio reported on the vaccine efficacy scale. The $x$-axis is the geometric mean concentration (GMC) of spike-specific IgG antibody binding measured by MSD and calibrated to the WHO standard (binding antibody units per $\mathrm{mL}$ ). Error bars indicate $95 \%$ confidence intervals for either the GMC IgG level (x-axis) or VE (y-axis). Weighted least-squares linear regression fit using inverse variance weighting on VE estimates (dashed line). Rank correlation coefficient, variance explained by the model, and mean squared error (MSE) are indicated for the Original and Alpha models. 
A

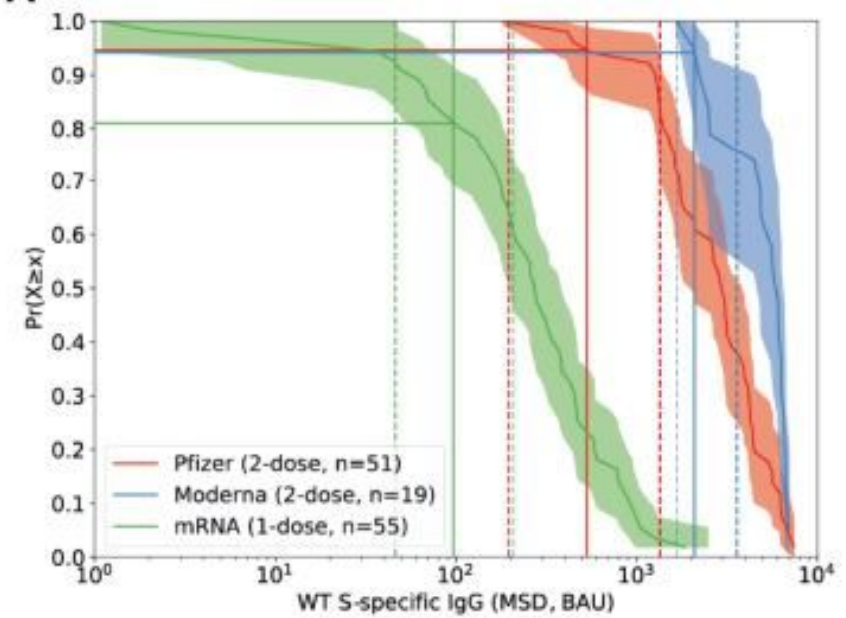

B

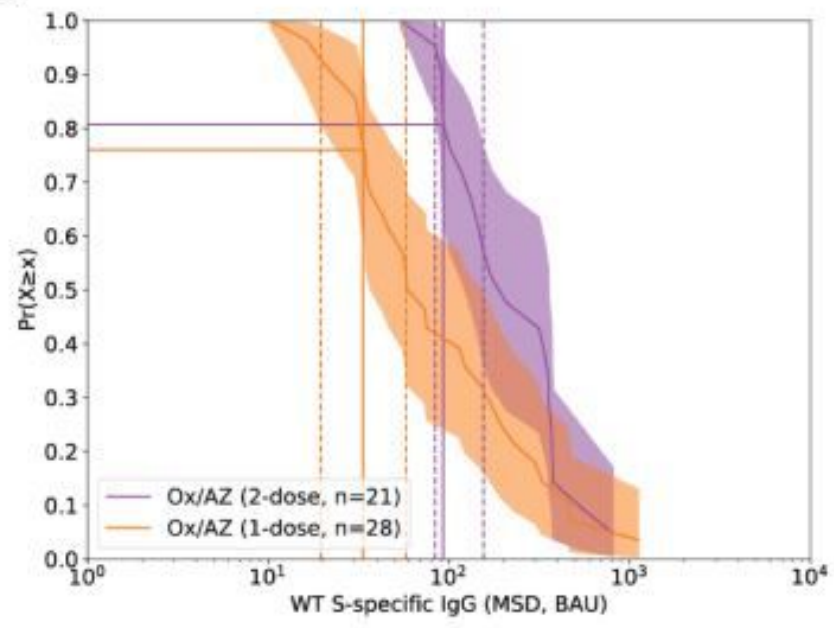

C

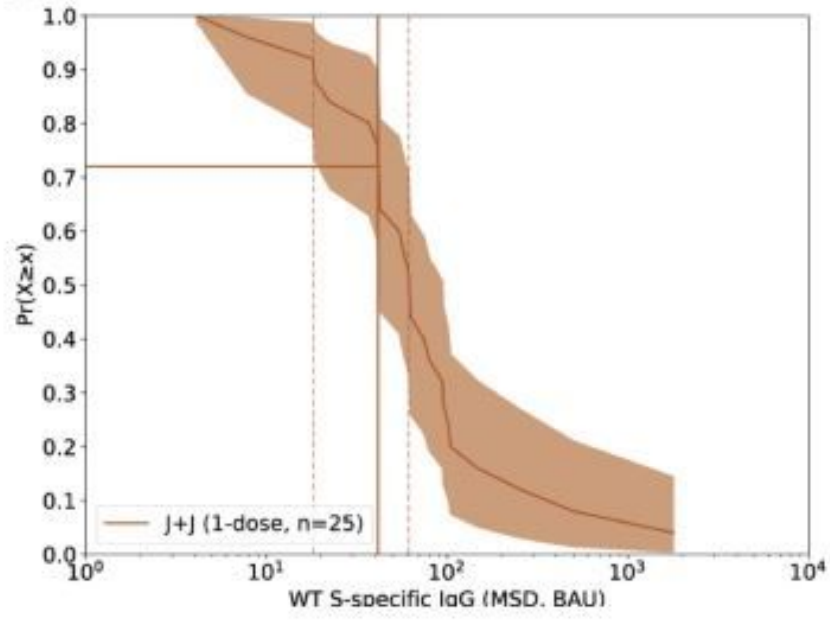

Figure 4

Distribution of spike-specific binding antibody vaccine responses and determination of a vaccine-specific protective threshold. Reverse cumulative distribution functions (RCDs) were estimated from spike-specific IgG vaccine responses measured in cohorts of individuals who received: (A) one or two doses of the Pfizer or Moderna mRNA-based vaccines, (B) one or two doses of the Oxford/AstraZeneca vaccine, (C) one dose of the $\mathrm{J}+\mathrm{J}$ vaccine. Each RCD along the y-axis represents the estimated proportion of 
participants who responded with at least as high a response as indicated along the $\mathrm{x}$-axis. Shaded region indicates a point-wise $95 \%$ confidence interval (Cl; see Methods for details). For each vaccine regimen, a published estimate of vaccine efficacy or effectiveness was used to compute a protective threshold by finding the VEth percentile of the RCD (solid horizontal line at VEth percentile). The protective threshold is shown (vertical sold line) with a $95 \% \mathrm{Cl}$ (dashed vertical line) that takes into account uncertainty in estimation of both the RCD and VE (see Methods for details).

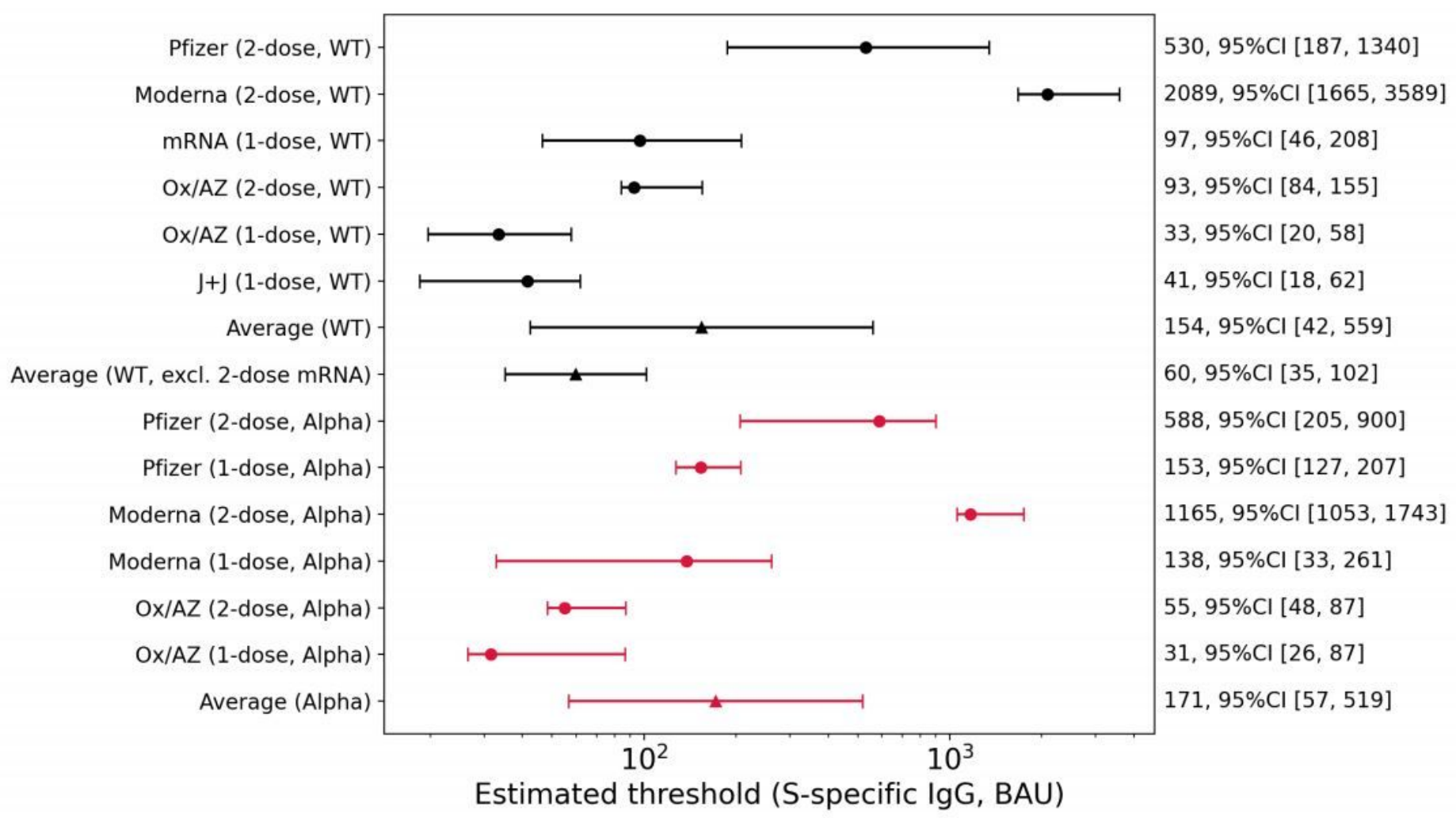

\section{Figure 5}

Summary of protective antibody binding thresholds derived for each vaccine. A protective threshold $(\mathrm{BAU} / \mathrm{ml})$ for each vaccine regimen is displayed with $95 \%$ confidence interval (circle symbols). Thresholds were computed both for original (WT, black) and alpha (red) spike-specific responses paired with WT and alpha virus-specific estimates of vaccine efficacy, respectively. An average overall protective threshold was computed for WT and alpha independently (triangle symbols) using a random effects meta-analytic approach (see Methods for details). Separately an average was computed for WT excluding the two-dose mRNA vaccine regimens. The protective threshold and $95 \% \mathrm{Cl}$ are annotated along the right $y$-axis.

\section{Supplementary Files}

This is a list of supplementary files associated with this preprint. Click to download. 
- GoldblattFinalSupplementary210821.docx 